ANALELE ŞTIINŢIFICE ALE UNIVERSITĂŢII “AL.I. CUZA” DIN IAŞI (S.N.)

MATEMATICA, Tomul LXI, 2015, f.1

DOI: $10.2478 /$ aicu-2014-0003

\title{
A MULTI-VALUED CHOQUET INTEGRAL WITH RESPECT TO A MULTISUBMEASURE
}

\author{
BY
}

FLOAREA-NICOLETA SOFIAN-BOCA

\begin{abstract}
JANG, KIM and KWON introduced a multi-valued Choquet integral for multifunctions with respect to real fuzzy measures and ZHANG, GUO and LIU established for this kind of integral some convergence theorems. The aim of this paper is to present another type of set-valued Choquet integral, called by us the Aumann-Choquet integral, for non-negative measurable functions with respect to multisubmeasures taking values in the class of all non-empty,compact and convex sets of $\mathbb{R}_{+}$on which we use the order relation considered by GuO and ZHANG. For this kind of integral, we study some important properties and we prove that if we add some supplementary properties to the multisubmeasure then they are also preserved by the set-valued function defined as Aumann-Choquet integral.
\end{abstract}

Mathematics Subject Classification 2010: 28B20, 28C15, $49 \mathrm{~J} 53$.

Key words: real Choquet integral, set-valued Choquet integral, multisubmeasure, absolute continuity.

\section{Introduction}

In recent years, there have appeared numerous works concerning Choquet integral which has drawn much attention due to the multiple applications in mathematics, economics, theory of control, decision making, risk analysis and many other fields.

Motivated by some problems in potential theory, in 1953/1954, CHOQUET ([2]) had been introduced an integral for non-negative measurable functions with respect to non-additive measures which has the monotonicity and continuity properties on certain sequences of sets. Later, the theory of Choquet integral was extended for the fuzzy measure, that is monotonic 
set functions. For this kind of Choquet integral was proved several important properties such as monotonicity and comonotonical additivity. Other properties of this integral was obtained under supplementary conditions imposed to the fuzzy measures $([14,15,21])$.

Using an Aumann type procedure ([1]), JANG ET AL. [10] introduced the concept of set-valued Choquet integral for a multifunction taking values in the class of all closed, nonempty sets of $\mathbb{R}_{+}$with respect to a nonegative fuzzy measure,for which the selectors are real Choquet integrals. For this integral, in [9], [10], [11] were obtained some properties and in [8] were proved some convergence theorems for sequences of multifunctions taking values in the class of all closed, nonempty sets of $\mathbb{R}_{+}$with respect to autocontinuous fuzzy measures, under Hausdorff convergence.

In [22], using the Kuratowski convergence, Zhang, GuO and LiU studied some properties of the set-valued Choquet integral of multifunctions with respect to real fuzzy measures and proved some convergence theorems for this kind of integral.

In [4], Hongxia and Jun proved that if are added supplementary properties to the fuzzy measures then they are also preserved by the multifunctions defined as the set-valued Choquet integral. In [6], [7] JANG presented some applications of Choquet integral for closed bounded interval-valued multifunctions.

Using the Aumann procedure, PRECUPANU and SATCO introduced and studied in [19], a set-valued integral of real functions with respect to finite additive multimeasures for which the selectors are Gould integrals with respect to finite additive measures taking their values in a Banach space (also see [17], [18]).

Based on analogous construction as in [19], we present in this paper a new type of set-valued Choquet integral for non-negative measurable functions with respect to multisubmeasures taking values in the class of all nonempty, compact and convex sets of $\mathbb{R}_{+}$.

The organisation of the paper is as follows: in section 2 we present notations and some basic concepts and in section 3 we recall some definitions and basic properties of classical Choquet integral of non-negative functions with respect to fuzzy measures ([14], [15], [16]). In section 4 we define another set-valued Choquet integral for non-negative bounded measurable functions with respect to multisubmeasures taking values in the class of all nonempty, compact and convex sets of $\mathbb{R}_{+}$, on which we use an order relation considered by GUO and ZHANG in [3]. We also present some remarkable 
properties of this kind of integral such as homogenity, monotonicity, linearity under some special conditions. In section 5, we proved that under supplementary conditions for the multisubmeasure such as null-additivity, converse null-additivity, pseudometric generating property or Darboux property then these are preserved by the corresponding Aumann Choquet integral.

\section{Terminology and notations}

Let $\mathrm{S}$ be a nonempty set, $\mathcal{A}$ an algebra of subsets of $S$ and $X=[0,+\infty)=$ $\mathbb{R}_{+}$.

We denote by: $\mathcal{P}_{0}(X)$, the family of all nonempty subsets of $X, \mathcal{P}_{k}(X)$, the family of all nonempty compact subsets of $X, \mathcal{P}_{f}(X)$, the family of all nonempty, closed subsets of $X, \mathcal{P}_{b}(X)$, the family of all nonempty, bounded subsets of $X, \mathcal{P}_{b f}(X)$, the family of all nonempty, bounded and closed subsets of $X, \mathcal{P}_{k c}(X)$, the family of nonempty, compact convex subsets of $X$.

For every $A, B \in \mathcal{P}_{0}(X)$ we denote by $e(A, B)=\sup _{x \in A} \inf _{y \in B}|x-y|$ and by $h: \mathcal{P}_{0}(Y) \times \mathcal{P}_{0}(Y) \rightarrow \mathbb{R}_{+}$the Hausdorff pseudometric defined by $h(A, B)=\max \{e(A, B), e(B, A)\}$.

We observe that $h$ becomes a metric on $\mathcal{P}_{b f}(X)$ ([5], ch.I.1, [13]).

We also denote by $|A|=h(A,\{0\})$, for every $A \in \mathcal{P}_{0}(X)$. By the definition of the Hausdorff metric, we have immediately the following:

Lemma 2.1. For every $A, B \in P_{k c}\left(\mathbb{R}_{+}\right)$, with $A=[a, b], B=[c, d]$ we have $h(A, B)=\max \{|a-c|,|b-d|\}$.

In [3], GuO and ZHANG consider the following order relation on $\mathcal{P}_{k c}(X)$ :

Definition 2.2. Let $A, B \in \mathcal{P}_{0}(X)$.

1) $A \lesssim B$ if:

(i) for each $x_{0} \in A$, there exists $y_{0} \in B$ such that $x_{0} \leqslant y_{0}$;

(ii) for each $y_{0} \in B$ there exists $x_{0} \in A$ such that $x_{0} \leqslant y_{0}$.

2) $A \lesssim B$ if:

(i') for each $x_{0} \in A$, there exists $y_{0} \in B$ such that $x_{0}<y_{0}$;

(ii') for each $y_{0} \in B$ there exists $x_{0} \in A$ such that $x_{0}<y_{0}$. 
It is easy to prove the following:

Proposition 2.3. If $A, B \in P_{k c}\left(\mathbb{R}_{+}\right)$where $A=[a, b]$ and $B=[c, d]$, $a, b, c, d \in \mathbb{R}_{+}$, we have $A \lesssim B$ if and only if $a \leq c$ and $b \leq d$.

Remark 2.4. 1) If $A, B \in \mathcal{P}_{k c}\left(\mathbb{R}_{+}\right)$, where $A=[a, b]$ and $B=[c, d]$, $a, b, c, d \in \mathbb{R}_{+}$, then $A \subseteq B$ means $c \leq a \leq b \leq d$.

2 ) It is easy to see that if $\mathrm{A}$ is a singleton, $A \in \mathcal{P}_{k c}\left(\mathbb{R}_{+}\right)$, the relation $\lesssim$ becomes the usual order relation on $\mathbb{R}_{+}$.

Definition $2.5([5])$. For a net $\left(A_{i}\right)_{i \in I} \subset \mathcal{P}_{0}\left(\mathbb{R}_{+}\right)$, where $I$ is a filtering set, we define $\lim \sup _{i \in I} A_{i}=\left\{x \in \mathbb{R}_{+}: x=\lim _{k} x_{i_{k}}, x_{i_{k}} \in A_{i_{k}}\right\}$ and $\liminf _{i \in I} A_{i}=\left\{x \in \mathbb{R}_{+}: x=\lim _{i} x_{i}, x_{i} \in A_{i}\right\}$.

We say that $A$ is the Kuratowski limit (briefly $K$-limit) of $\left(A_{i}\right)$ if $\limsup _{i \in I} A_{i}=\liminf _{i \in I} A_{i}=A$ and we denote it by $\mathrm{A}_{i} \stackrel{K}{\rightarrow} A$ (or, simply $\mathrm{A}_{i} \rightarrow A$ ).

This kind of convergence is called Kuratowski convergence (briefly $K$ convergence).

Lemma 2.6 ([23]). If $A_{i}=\left[a_{i}, b_{i}\right] \subset P_{k c}\left(\mathbb{R}_{+}\right), i \in I$, then $A_{i} \stackrel{K}{\rightarrow} A=$ $[a, b]$ if and only if $a_{i} \rightarrow a, b_{i} \rightarrow b$.

Definition $2.7([22])$. Let $\left(A_{n}\right)_{n \in \mathbb{N}} \subset \mathcal{P}_{f}\left(\mathbb{R}_{+}\right)$be a sequence and $A \in$ $\mathcal{P}_{f}\left(\mathbb{R}_{+}\right)$. We say that:

1) $\left(A_{n}\right)$ is increasing $K$-convergent to $A$ if $A_{1} \lesssim A_{2} \lesssim \ldots$ and $A_{n} \stackrel{K}{\rightarrow} A$ and we denote it by $A_{n} \uparrow A$;

2) $\left(A_{n}\right)$ is decreasing $K$-convergent to $A$ if $A_{1} \gtrsim A_{2} \gtrsim \ldots$ and $A_{n} \stackrel{K}{\rightarrow} A$ and we denote it by $A_{n} \downarrow A$.

Remark 2.8. From [5], ch.1, we have that if $\left(A_{i}\right)_{i \in I} \subset \mathcal{P}_{k c}(\mathbb{R})$ then $K$ convergence of $\left(A_{i}\right)_{i \in I}$ is equivalent to its convergence in Hausdorff metric. It is easy to prove that the same is true for $\mathcal{P}_{k c}\left(\mathbb{R}_{+}\right)$.

Definition 2.9 ([7]). If $[a, b],[c, d] \in \mathcal{P}_{k c}\left(\mathbb{R}_{+}\right)$and $k \in \mathbb{R}_{+}$, then we define:

$$
\begin{aligned}
& {[a, b]+[c, d]=[a+c, b+d],} \\
& k[a, b]=[k a, k b], \\
& {[a, b][c, d]=[a c, b d],} \\
& {[a, b] \wedge[c, d]=[a \wedge c, b \wedge d] \text { and }[a, b] \vee[c, d]=[a \vee c, b \vee d],}
\end{aligned}
$$

where $a \wedge b=\min \{a, b\}$ and $a \vee b=\min \{a, b\}$. 
Remark 2.10. For all $A, B \in \mathcal{P}_{k c}\left(\mathbb{R}_{+}\right)$we have:

i) $A \lesssim A \vee B$ and $B \lesssim A \vee B$

ii) $A \wedge B \lesssim A$ and $A \wedge B \lesssim B$.

Definition 2.11 ([14]). Let be $f, g: S \rightarrow \mathbb{R}$ two functions. We say that $f$ and $g$ are comonotonic and we denote it by $f \sim g$, if $f(s)<f\left(s^{\prime}\right) \Rightarrow$ $g(s) \leq g\left(s^{\prime}\right)$, for every $s, s^{\prime} \in S$.

Definition 2.12. The set function $m: \mathcal{A} \rightarrow \mathbb{R}_{+}$with $\mathrm{m}(\emptyset)=0$ is said to be:

1) a fuzzy measure if $m(A) \leq m(B)$, for every $A, B \in \mathcal{A}$ with $A \subseteq B$;

2) a submeasure in Drewnowski sense if:

(i) $m(A \cup B) \leq m(A)+m(B)$, for every $A, B \in \mathcal{A}$ with $A \cap B=\emptyset$;

(ii) $m(A) \leq m(B)$, for every $A, B \in \mathcal{A}$ with $A \subseteq B$.

3) a strict submeasure if:

(i') $m(A \cup B)<m(A)+m(B)$, for every $A, B \in \mathcal{A}$ with $A \cap B=\emptyset$;

(ii') $m(A)<m(B)$, for every $A, B \in \mathcal{A}$ with $A \subseteq B$.

4) an additive measure (shortly a measure) if $m(A \cup B)=m(A)+m(B)$, for every $A, B \in \mathcal{A}$ with $A \cap B=\emptyset$.

The order in the Definition 2.12. is the usual order on $\mathbb{R}_{+}$.

Definition 2.13. A set-valued function $\mu: \mathcal{A} \rightarrow \mathcal{P}_{k c}\left(\mathbb{R}_{+}\right)$with $\mu(\emptyset)=$ $\{0\}$ is said to be:

1) a fuzzy multimeasure if $\mu(A) \subseteq \mu(B)$, for every $A, B \in \mathcal{A}$ with $A \subseteq B$;

2) a monotone set-function if $\mu(A) \lesssim \mu(B)$, for every $A, B \in \mathcal{A}$ with $A \subseteq B$

3) a multisubmeasure if the following conditions are satisfied:

(i) $\mu(A \cup B) \lesssim \mu(A)+\mu(B)$, for every $A, B \in \mathcal{A}$ with $A \cap B=\emptyset$;

(ii) $\mu(A) \lesssim \mu(B)$, for every $A, B \in \mathcal{A}$ with $A \subseteq B$; 
4) a strict multisubmeasure if:

(i') $\mu(A \cup B) \lesssim \mu(A)+\mu(B)$, for every $A, B \in \mathcal{A}$ with $A \cap B=\emptyset$;

(ii') $\mu(A) \lesssim \mu(B)$, for every $A, B \in \mathcal{A}$ with $A \subseteq B$.

5) an additive multimeasure (shortly a multimeasure) if $\mu(A \cup B)=$ $\mu(A) \dot{+} \mu(B)$, for every $A, B \in \mathcal{A}$ with $A \cap B=\emptyset$, where we denoted by $E+F=\overline{E+F}, \forall E, F \in \mathcal{A}$ in which $\bar{E}$ means the closure of $E$.

Unless stated otherwise, in what follows we consider that $\mu: \mathcal{A} \rightarrow$ $\mathcal{P}_{k c}(X)$ is a multisubmeasure.

Definition 2.14 ([4]). 1) The set multifunction $\mu: \mathcal{A} \rightarrow \mathcal{P}_{0}(X)$ is called:

i) null-additive if for every $A, B \in \mathcal{A}$ with $\mu(A)=\{0\}$ we have $\mu(A \cup$ $B)=\mu(B)$

ii) weakly null-additive if for every $A, B \in \mathcal{A}$ with $\mu(A)=\mu(B)=\{0\}$ we have $\mu(A \cup B)=\{0\}$;

iii) converse null-additive if for every $A, B \in \mathcal{A}$ with $A \subset B$ such that $\mu(A)=\mu(B)$ we have $\mu(B-A)=\{0\} ;$

iv) order continuous (briefly o-continuous) if for every $\left(A_{n}\right)_{n \in \mathbb{N}} \subset \mathcal{A}, A_{n} \downarrow$ $A$ with $\mu(A)=\{0\}$ we have $\lim _{n \rightarrow \infty} \mu\left(A_{n}\right)=\{0\}$;

v) lower semi-continuous if for $\left(A_{n}\right)_{n \in \mathbb{N}} \subset \mathcal{A}, A_{n} \uparrow A$ we have $h\left(\mu\left(A_{n}\right)\right.$, $\mu(A)) \rightarrow 0$.

Definition 2.15. The set multifunction $\mu: \mathcal{A} \rightarrow \mathcal{P}_{0}(X)$ is said to have:

i) the pseudometric generating property (briefly p.g.p.) if for every $\varepsilon>0$ there exists $\delta>0$ such that for $A, B \in \mathcal{A}$ with $\mu(A) \subset[0, \delta], \mu(B) \subset$ $[0, \delta]$ we have $\mu(A \cup B) \subset[0, \varepsilon]$;

ii) the Darboux property (briefly d.p.) if for every $A \in \mathcal{A}$ with $\mu(A) \supsetneq$ $\{0\}$, there exists $p \in(0,1)$ and a set $B \in \mathcal{A}$ such that $B \subset A$ and $\mu(B)=p \mu(A)$.

iii) the property $(S)$ if for any $\left(A_{n}\right)_{n \in \mathbb{N}} \subset \mathcal{A}$ such that $\lim _{n \rightarrow \infty} \mu\left(A_{n}\right)=\{0\}$ there exists a subsequence $\left(A_{n_{k}}\right) \subset\left(A_{n}\right)$ such that $\mu\left(\liminf _{k \rightarrow \infty} A_{n_{k}}\right)$ $=\{0\}$. 
Definition $2.16([4])$. Let be $\mu: \mathcal{A} \rightarrow \mathcal{P}_{k c}\left(\mathbb{R}_{+}\right)$a multisubmeasure and $\lambda: \mathcal{A} \rightarrow \mathbb{R}_{+}$a fuzzy measure. We say that:

1) $\mu$ is absolutely continuous of type $I$ with respect to $\lambda$, denoted by $\mu \ll_{I} \lambda$, if for every $A \in \mathcal{A}$ with $\lambda(A)=0$ we have $\mu(A)=\{0\}$;

2) $\mu$ is absolutely continuous of type $I I$ with respect to $\lambda$, denoted by $\mu \ll_{I I} \lambda$, if for every $B \subset A, A, B \in \mathcal{A}$ for which $\lambda(A-B)=0$ we have $\mu(A)=\mu(B)$;

3) $\mu$ is absolutely continuous of type III with respect to $\lambda$, denoted by $\mu \ll_{I I I} \lambda$, if for every $\left(A_{n}\right)_{n \in \mathbb{N}} \subset \mathcal{A}$ with $\lim _{n \rightarrow \infty} \lambda\left(A_{n}\right)=0$ we have $\lim _{n \rightarrow \infty} \mu\left(A_{n}\right)=\{0\}$;

4) $\mu$ is absolutely continuous of type $I V$ with respect to $\lambda$, denoted by $\mu \ll_{I V} \lambda$, if for every $\varepsilon>0$ there exists $\delta>0$ such that for any $\mathrm{A} \in \mathcal{A}$ with $\lambda(A) \leq \delta$ we have $\mu(A) \subseteq[0, \varepsilon]$.

Remark 2.17. If $\mu: \mathcal{A} \rightarrow \mathcal{P}_{k c}\left(\mathbb{R}_{+}\right)$is a null-additive multisubmeasure and $\lambda$ is a fuzzy measure we have that $\mu \ll_{I} \lambda \Leftrightarrow \mu \ll_{I I} \lambda$.

Indeed, let be $\mu \ll_{I} \lambda$ and $B \subset A, \mathcal{A} \in \mathcal{A}$, with $\lambda(A-B)=0$. Then from $\mu \ll_{I} \lambda$ we obtain that $\mu(A-B)=\{0\}$. Because $\mu$ is a multisubmeasure null-additive we have that $\mu(A)=\mu((A-B) \cup B)=\mu(B)$ which assures that $\mu \ll_{I I} \lambda$.

Conversely, let be $\mu \ll_{I I} \lambda$ and $A \in \mathcal{A}$ with $\lambda(A)=0$. If we particularly take $B=\emptyset, B \subset A$ then $\lambda(A-B)=\lambda(A)=0$. Since $\mu \ll_{I I} \lambda$ we have that $\mu(A)=\mu(B)=\{0\}$, so $\mu \ll_{I} \lambda$.

Definition 2.18. We say that a property $P$ holds $\mu$-almost everywhere ( $\mu$-a.e.) on $S$ if the property $P$ is true on $S-N$, with $\mu(N)=\{0\}$.

Definition 2.19. Let be a set multifunction $\mu: \mathcal{A} \rightarrow \mathcal{P}_{0}\left(\mathbb{R}_{+}\right)$. The set function $m: \mathcal{A} \rightarrow X$ is a selector of $\mu$ if for every $A \in \mathcal{A}$ we have $m(A) \in \mu(A)$.

We denote by $\mathrm{S}_{\mu}$ the set of all selectors of the set multifunction $\mu$.

Remark 2.20. If $\mu: \mathcal{A} \rightarrow \mathcal{P}_{k c}\left(\mathbb{R}_{+}\right)$is a multisubmeasure, we can associate to $\mu$ two nonnegative set functions:

$$
m_{1}(A)=\inf \mu(A), \forall A \in \mathcal{A} \text { and } m_{2}(A)=\sup \mu(A), \text { for every } A \in \mathcal{A} \text {. }
$$


According to [23], Proposition 2.1, $m_{1}, m_{2}$ are submeasures in Drewnowski sense, such that $m_{1}(A) \in \mu(A)$ and $m_{2}(A) \in \mu(A)$, for every $A \in$ $\mathcal{A}$. Thus, every multisubmeasure has selectors that are fuzzy measures. Moreover, for $\mu: \mathcal{A} \rightarrow \mathcal{P}_{k c}\left(\mathbb{R}_{+}\right)$we have that $\mu(A)=\left[m_{1}(A), m_{2}(A)\right]$, for every $A \in \mathcal{A}$.

In what follows we shall also use:

Lemma 2.21. If $A, B, C, D \in P_{k c}\left(\mathbb{R}_{+}\right)$with $A \subseteq B, C \subseteq D$ and $B \lesssim D$ then $A \lesssim C$.

Proof. Since $A, B, C, D \in \mathcal{P}_{k c}\left(\mathbb{R}_{+}\right)$then we can write $A=\left[a_{1}, a_{2}\right], B=$ $\left[b_{1}, b_{2}\right], C=\left[c_{1}, c_{2}\right], D=\left[d_{1}, d_{2}\right]$, where $a_{1}, a_{2}, b_{1}, b_{2}, c_{1}, c_{2}, d_{1}, d_{2} \in \mathbb{R}_{+}$. From $A \subseteq B$ and $C \subseteq D$ we have $b_{1} \leq a_{1} \leq a_{2} \leq b_{2}$ and, respectively, $d_{1} \leq c_{1} \leq c_{2} \leq d_{2}$.

Since $B \lesssim D$ we obtain $b_{1} \leq d_{1}$ and $b_{2} \leq d_{2}$.

We have the following cases:

a) $b_{1} \leq a_{1} \leq d_{1} \leq c_{1} \leq a_{2} \leq b_{2} \leq c_{2} \leq d_{2}$;

b) $b_{1} \leq d_{1} \leq a_{1} \leq a_{2} \leq b_{2} \leq c_{1} \leq c_{2} \leq d_{2}$ and respectively,

c) $b_{1} \leq a_{1} \leq a_{2} \leq d_{1} \leq c_{1} \leq b_{2} \leq c_{2} \leq d_{2}$.

It is easy to see that in all cases, we obtain that $a_{1} \leq c_{1}$ and $a_{2} \leq c_{2}$, so $A \lesssim C$.

Definition $2.22([12])$. Let be $\mu, \nu: A \rightarrow P_{k c}\left(\mathbb{R}_{+}\right)$two multisubmeasures. We define, for every $A \in \mathcal{A},(\mu \vee \nu)(A)=\mu(A) \vee \nu(A)$ and $(\mu \wedge \nu)(A)=$ $\mu(A) \wedge \nu(A)$.

\section{The Choquet integral}

In this section we recall the definition and some basic properties of the real-valued Choquet integral.

Let be $f: S \rightarrow \mathbb{R}_{+}$a measurable function and $m: \mathcal{A} \rightarrow \mathbb{R}_{+}$a fuzzy measure.

Definition 3.1 ([14, 15, 16]). 1) The Choquet integral of the function $f$ with respect to the fuzzy measure $m$ on $A \in \mathcal{A}$ is defined by

$$
\text { (C) } \int_{A} f d m=\int_{0}^{\infty} m(\{s: f(s) \geq t\} \cap A) d t,
$$


where the integral on the right-hand is the Lebesgue integral.

2) The function $f$ is called Choquet integrable on $A$ if $(C) \int_{A} f d m<\infty$.

If $A=S$ then we write $(C) \int_{S} f d m$ or $(C) \int f d m$. We denote by $\mathcal{L}_{C}(m, A)$ the set of all Choquet integrable functions on $A \in \mathcal{A}$ and by $\mathcal{L}_{C}(m, S)$ (or shortly $\mathcal{L}_{C}(m)$ ) the set of all Choquet integrable functions on $S$.

We mention the following properties for Choquet integral defined by (2):

Theorem $3.2([14,15,16])$. Let be $m: \mathcal{A} \rightarrow \mathbb{R}_{+}$a fuzzy measure and $f, g: S \rightarrow \mathbb{R}_{+}$Choquet integrable functions on $S$. The following statements hold:

i) if $f=0$ then $(C) \int f d m=0$;

ii) for every $A \in \mathcal{A},(C) \int \chi_{A} d m=m(A)$, where $\chi_{A}$ is the characteristic function of the set $A$;

iii) for any $\alpha \geq 0$ we have $(C) \int \alpha f d m=\alpha(C) \int f d m$;

iv) if $\alpha>0, \alpha m$ is a fuzzy measure and $(C) \int f d(\alpha m)=\alpha(C) \int f d m$;

$v)$ if $m(S)<\infty$ then for every $\alpha \in \mathbb{R}_{+}$we have $(C) \int(f+\alpha) d m=$ (C) $\int f d m+\alpha m(S)$;

vi) for every $A, B \in \mathcal{A}$ with $A \subset B, f$ is Choquet integrable on $A$ and $B$ and we have $(C) \int_{A} f d m \leq(C) \int_{B} f d m$.

Theorem $3.3([14,15,16])$. Let be $m: \mathcal{A} \rightarrow \mathbb{R}_{+}$a fuzzy measure and $f, g: S \rightarrow \mathbb{R}_{+}$Choquet integrable functions on $S$.

If $f \leq g$ on $S$, then $(C) \int f d m \leq(C) \int g d m$.

Proposition $3.4([14])$. Let be $m: \mathcal{A} \rightarrow \mathbb{R}_{+}$a fuzzy measure and $f, g:$ $S \rightarrow \mathbb{R}_{+}$Choquet integrable functions on $S$. Then the following statements hold:

i) $(C) \int(f \vee g) d m \geq(C) \int f d m \vee(C) \int g d m$ and $(C) \int(f \wedge g) d m \leq$ $(C) \int f d m \wedge(C) \int g d m$, where $(f \vee g)(s)=\max \{f(s), g(s)\}$ and $(f \wedge$ $g)(s)=\min \{f(s), g(s)\}$, for all $s \in S$;

ii) for every $A, B \in A$ we have $(C) \int_{A \cap B} f d m \leq(C) \int_{A} f d m \wedge(C) \int_{B} f d m$ and $(C) \int_{A \cup B} f d m \geq(C) \int_{A} f d m \vee(C) \int_{B} f d m$. 
Proposition $3.5([12,14])$. Let be $m, n: \mathcal{A} \rightarrow \mathbb{R}_{+}$two fuzzy measures such that $m(S)=n(S)$ and $f: S \rightarrow \mathbb{R}_{+}$a measurable function, Choquet integrable on $S$ with respect to $m$ and $n$. Then:

i) $(C) \int f d(m+n)=(C) \int f d m+(C) \int f d n$;

ii) if $m \leq n$ then $(C) \int f d m \leq(C) \int f d n$.

One of the most important property of Choquet integral is the comonotonic additivity that is the Choquet integral is additive for functions which are comonotone.

Theorem 3.6 ([16]). Let be $m: \mathcal{A} \rightarrow \mathbb{R}_{+}$a fuzzy measure and $f, g:$ $S \rightarrow \mathbb{R}_{+}$measurable functions Choquet integrable on $S$ such that $f, g$ are comonotonic on $S$. Then $(C) \int(f+g) d m=(C) \int f d m+(C) \int g d m$.

\section{A multi-valued Choquet integral with respect to a multi- submeasure}

In [10], JANG ET AL. introduced a multi-valued Choquet integral for a multifunction taking values in the class of all closed, nonempty sets of $\mathbb{R}_{+}$ with respect to a nonegative fuzzy measure, for which the selectors are real Choquet integrals and in [6], [7] JANG presented some properties of the setvalued Choquet integral for closed bounded interval-valued multifunctions.

In this section, we introduce and study another multivalued Choquet integral, this time for non-negative functions with respect to multisubmeasures taking values in $\mathcal{P}_{k c}\left(\mathbb{R}_{+}\right)$.

In what follows we consider that $\mu: \mathcal{A} \rightarrow \mathcal{P}_{k c}\left(\mathbb{R}_{+}\right)$is a multisubmeasure.

From Remark 2.20 we have that $\mu(A)=\left[m_{1}(A), m_{2}(A)\right]$, for every $A \in$ $\mathcal{A}$,and $S_{\mu} \neq \emptyset$ because contains the submeasures $m_{1}$ and $m_{2}$. Let be $f$ : $S \rightarrow \mathbb{R}_{+}$a measurable function. We denote by $S_{\mu}^{C}(f)$ or $S_{\mu}^{C}$ the set of all fuzzy measures $m: \mathcal{A} \rightarrow \mathbb{R}_{+}$which are selectors of the multisubmeasure $\mu$ with respect to which the function $\mathrm{f}$ is Choquet integrable. Using a similar procedure as in [19] for Aumann-Gould integral we give:

Definition 4.1. Let be $\mu: \mathcal{A} \rightarrow \mathcal{P}_{k c}\left(\mathbb{R}_{+}\right)$a multisubmeasure, $f: S \rightarrow$ $\mathbb{R}_{+}$a measurable function and $A \in \mathcal{A}$.

1) The Aumann-Choquet integral (shortly $(A C)$-integral) of $f$ on $A$ with respect to the multisubmeasure $\mu$ is the set

$$
(A C) \int_{A} f d \mu=\left\{(C) \int_{A} f d m, m \in S_{\mu}^{C}(f)\right\},
$$


where $(C) \int_{A} f d m$ is the Choquet integral defined by (2).

2) The function $f$ is said to be Aumann-Choquet integrable on $A$ if $(A C) \int_{A} f d \mu \neq \emptyset$.

If $A=S$ we denote the integral by $(A C) \int_{S} f d \mu$ or simply $(A C) \int f d \mu$.

We also denote by $\mathcal{L}_{A C}(\mu, A)$ the set of all Aumann-Choquet integrable functions with respect to $\mu$ on $A$ and by $\mathcal{L}_{A C}(\mu, S)$ or $\mathcal{L}_{A C}(\mu)$ the set of all Aumann-Choquet integrable functions with respect to $\mu$ on $S$.

Proposition 4.2. Let be $\mu: \mathcal{A} \rightarrow \mathcal{P}_{k c}\left(\mathbb{R}_{+}\right)$a multisubmeasure and $f: S \rightarrow \mathbb{R}_{+}$a measurable function such that $f \in \mathcal{L}_{C}\left(m_{2}\right)$, where $m_{2}$ is defined by (1). Then the function $f$ is $A C$-integrable on every $A \in \mathcal{A}$.

Proof. From Remark 2.20 we have that $\mu(A)=\left[m_{1}(A), m_{2}(A)\right]$, for every $A \in \mathcal{A}$, and since $f \in \mathcal{L}_{C}\left(m_{2}\right)$, then $f \in \mathcal{L}_{C}\left(m_{2}, A\right)$. Hence, from Definition 3.1,2), we have that $(C) \int_{A} f d m_{2}<\infty$. Then, for every $m \in$ $S_{\mu}(f)$ we have that $m(A) \leq m_{2}(A)$, for every $A \in \mathcal{A}$. From Theorem 3.5 , ii) we obtain $(C) \int_{A} f d m<(C) \int_{A} f d m_{2}<\infty$, which assures that $f \in \mathcal{L}_{A C}(\mu, A)$.

Proposition 4.3. Let be $\mu: \mathcal{A} \rightarrow \mathcal{P}_{k c}\left(\mathbb{R}_{+}\right)$a multisubmeasure and $f: S \rightarrow \mathbb{R}_{+}$an AC-integrable function on $S$. Then $S_{\mu}^{C}(f)$ is a convex set.

Proof. Let be $S_{\mu}^{C}(f)=\left\{m \in S_{\mu} ;(C) \int f d m<\infty\right\} \neq \emptyset$ and $m, n \in$ $S_{\mu}^{C}(f)$. Let be $\alpha \in(0,1)$. Since $m \in S_{\mu}^{C}(f)$ we have $(C) \int f d m<\infty$, and from $n \in S_{\mu}^{C}(f)$ we have $(C) \int f d n<\infty$. If we denote by $\nu=\alpha m+(1-\alpha) n$ then, evidently, $\nu \in S_{\mu}$. According to Theorems 3.2, iv) and 3.5 we obtain $(A C) \int f d \nu=(C) \int f d(\alpha m+(1-\alpha) n)=\alpha(C) \int f d m+(1-\alpha)(C) \int f d n<$ $\infty$ and, consequently $\nu=\alpha m+(1-\alpha) n \in S_{\mu}^{C}(f)$ and hence $S_{\mu}^{C}(f)$ is a convex set.

In the following, we point out some immediate properties of the AumannChoquet integral.

Proposition 4.4. Let be $\mu: \mathcal{A} \rightarrow \mathcal{P}_{k c}\left(\mathbb{R}_{+}\right)$a multisubmeasure and $f: S \rightarrow \mathbb{R}_{+}$a measurable function. Then:

i) if $f=0$ on $S$ then $f$ is $A C$ integrable on $S$ and $(A C) \int f d \mu=\{0\}$;

ii) if $\mu(A)=\{0\}$ then $(A C) \int_{A} f d \mu=\{0\}$. 
Proof. i) Since $f=0$ on $S$ then for every $m \in S_{\mu}^{C}(f)$ and all $t \geq 0$ we have from Theorem 3.2, i) that $(C) \int f d m=\int_{0}^{\infty} m(\{s: f(s) \geq t\}) d t=0$ hence $(A C) \int f d \mu=\{0\}$.

For ii) let us observe that since $\mu(A)=\{0\}$ then from Remark 2.20 and $\mu(A) \in P_{k c}\left(\mathbb{R}_{+}\right)$we have that $\mu(A)=\left[m_{1}(A), m_{2}(A)\right]=\{0\}$ hence $m_{1}(A)=m_{2}(A)=0$. Then, for every $m \in S_{\mu}^{C}$, we have $m(A)=0$. Hence, $(C) \int_{A} f d m=\int_{0}^{\infty} m(\{s: f(s) \geq t\} \cap A) d t=0$ and we have that (AC) $\int_{A} f d \mu=\{0\}$.

Proposition 4.5. Let be $\mu: \mathcal{A} \rightarrow \mathcal{P}_{k c}\left(\mathbb{R}_{+}\right)$a multisubmeasure and $f, g: S \rightarrow \mathbb{R}_{+}$measurable functions, (AC)-integrable with respect to $\mu$ on $S$, such that $f=g(\mu-a . e)$. Then $(A C) \int f d \mu=(A C) \int g d \mu$.

Proof. Since $f, g \in \mathcal{L}_{A C}(\mu)$ then $(A C) \int f d \mu=\left\{(C) \int f d m, m \in\right.$ $\left.S_{\mu}^{C}(f)\right\} \neq \emptyset$ and $(A C) \int g d \mu=\left\{(C) \int g d m, m \in S_{\mu}^{C}(g)\right\} \neq \emptyset$. Let be any $x_{0} \in(A C) \int f d \mu$. Then there exists $m \in S_{\mu}^{C}(f)$ such that $x_{0}=(C) \int f d m$. Since $f=g(\mu$-a.e. $)$ then there exists $N \in \mathcal{A}$ with $\mu(N)=\{0\}$ such that $f(s)=g(s)$, for every $s \in c N$ and at the same time we have $f=g(m$-a.e. $)$.

Thus $x_{0}=(C) \int_{c N} f d m=(C) \int_{c N} g d m$ and consequently $(C) \int g d m \in$ $(A C) \int g d \mu$. Hence $(A C) \int f d \mu \subseteq(A C) \int g d \mu$.

Analogously, we can prove that $(A C) \int g d \mu \subseteq(A C) \int f d \mu$.

Proposition 4.6. Let be $\mu: \mathcal{A} \rightarrow \mathcal{P}_{k c}\left(\mathbb{R}_{+}\right)$a multisubmeasure and $f:$ $S \rightarrow \mathbb{R}_{+}$an AC-integrable function on $S$. Then $f$ is Aumann-Choquet integrable on every $E \in \mathcal{A}$.

Proof. Since $f \in \mathcal{L}_{A C}(\mu)$ we have that

$(A C) \int f d \mu=\left\{(C) \int f d m, m \in S_{\mu}^{C}(f)\right\} \neq \emptyset$. Let be any $y_{0} \in(A C) \int f d \mu$. Then there exists $m \in S_{\mu}^{C}(f)$ such that $y_{0}=(C) \int f d m<\infty$. Let be any $E \in \mathcal{A}$. We have, for all $t \geq 0$, that $\{s: f(s) \geq t\} \cap E \subset\{s: f(s) \geq t\}$ and since $m: \mathcal{A} \rightarrow \mathbb{R}_{+}$is a fuzzy measure selector of multisubmeasure $\mu$ we obtain that $m(\{s: f(s) \geq t\} \cap E) \leq m(\{s: f(s) \geq t\})$ and hence we have that (C) $\int_{E} f d m \leq(C) \int f d m<\infty$. Therefore $m \in S_{\mu}^{C}(f, E)$ and consequently, $(A C) \int_{E} f d \mu \neq \emptyset$, that is $f \in \mathcal{L}_{A C}(\mu, E)$.

Theorem 4.7. Let be $\mu: \mathcal{A} \rightarrow \mathcal{P}_{k c}\left(\mathbb{R}_{+}\right)$a multisubmeasure, $f: S \rightarrow \mathbb{R}_{+}$ an $A C$ - integrable function with respect to $\mu$ on $S$ and $\alpha>0$. The following statements hold:

i) $\alpha f$ is $A C$-integrable with respect to $\mu$ on $S$ and $(A C) \int(\alpha f) d \mu=$ $a(A C) \int f d \mu$; 
ii) $f$ is AC-integrable with respect to $\alpha \mu$ on $S$ and $(A C) \int f d(\alpha \mu)=$ $a(A C) \int f d \mu$.

Proof. i) From AC-integrability of $f$ on $S$ we have that $(A C) \int f d \mu=$ $\left\{(C) \int f d m, m \in S_{\mu}^{C}(f)\right\} \neq \emptyset$. Let be $y_{0} \in(A C) \int f d \mu$. Then there exists $m \in S_{\mu}^{C}(f)$ such that $y_{0}=(C) \int f d m<\infty$.

From Theorem 3.2, iii) we have that $(C) \int(\alpha f) d m=\alpha(C) \int f d m=$ $\alpha y_{0}$. Then $m \in S_{\mu}^{C}(\alpha f)$ and we obtain $(A C) \int(\alpha f) d \mu=\left\{(C) \int(\alpha f) d m\right.$, $\left.m \in S_{\mu}^{C}(\alpha f)\right\}=\left\{\alpha(C) \int f d m, m \in S_{\mu}^{C}(f)\right\}=\left\{\alpha y, y \in(A C) \int f d \mu\right\}=$ $a(A C) \int f d \mu$. Hence $(A C) \int(\alpha f) d \mu=a(A C) \int f d \mu$.

In order to prove ii), let us observe that we have $(A C) \int f d(\alpha \mu)=$ $\left\{(C) \int f d m, m \in S_{\alpha \mu}^{C}(f)\right\}$.

Since $f$ is AC-integrable, for any $z_{0} \in(A C) \int f d \mu$ there exists $n \in$ $S_{\mu}^{C}(f)$ such that $z_{0}=(C) \int f d n<\infty$. From Theorem 3.2, iv) we obtain that $(C) \int f d(\alpha n)=\alpha(C) \int f d n<\infty$. Hence $\alpha n=m^{\prime} \in S_{\alpha \mu}^{C}(f)$ and $(C) \int f d m^{\prime}<\infty$. Then $f \in \mathcal{L}_{A C}(\alpha \mu)$ and $(A C) \int f d(\alpha \mu)=\left\{(C) \int f d m^{\prime}\right.$, $\left.m^{\prime} \in S_{\alpha \mu}^{C}(f)\right\}=\left\{(C) \int f d(\alpha n), n \in S_{\mu}^{C}(f)\right\}=\left\{\alpha(C) \int f d n, n \in S_{\mu}^{C}(f)\right\}=$ $a(A C) \int f d \mu$.

Theorem 4.8. Let be $\mu, v: \mathcal{A} \rightarrow \mathcal{P}_{k c}\left(\mathbb{R}_{+}\right)$two multisubmeasures and $f:$ $S \rightarrow \mathbb{R}_{+}$a function such that $f \in \mathcal{L}_{A C}(\mu) \cap \mathcal{L}_{A C}(v)$. Then, for every $\alpha, \beta>$ $0, f$ is $A C$-integrable with respect to $\alpha \mu+\beta v$ on $S$ and $(A C) \int f d(\alpha \mu+\beta v)=$ $a(A C) \int f d \mu+\beta(A C) \int f d v$.

Proof. Let be any $\alpha, \beta>0$. From AC-integrability of $\mathrm{f}$ with respect to $\mu$, respectively to $v$, we have $(A C) \int f d \mu \neq \emptyset$ and $(A C) \int f d v \neq \emptyset$. From Theorem 4.7, ii) we have that $(A C) \int f d(\alpha \mu)=a(A C) \int f d \mu$, respectively $(A C) \int f d(\beta v)=\beta(A C) \int f d v$.

Let be $x_{1} \in(A C) \int f d(\alpha \mu)$ and $x_{2} \in(A C) \int f d(\beta v)$, arbitrarily chosen. Then there exists $m^{\prime} \in S_{\alpha \mu}^{C}(f)$ and $n^{\prime} \in S_{\beta v}^{C}(f)$ such that $x_{1}=(C) \int f d m^{\prime}$ and $x_{2}=(C) \int f d n^{\prime}$. Since $m^{\prime} \in S_{\alpha \mu}^{C}(f)$ and $n^{\prime} \in S_{\beta v}^{C}(f)$ then we obtain that $m^{\prime}=\alpha m$, where $m \in S_{\mu}^{C}(f)$ and, respectively, $n^{\prime}=\beta n$, where $n \in$ $S_{v}^{C}(f)$.

Using Theorem 3.2, iv) and Theorem 3.5, i) we have that $m^{\prime}+n^{\prime} \in$ $S_{\alpha \mu+\beta v}^{C}(f)$ and $(C) \int f d\left(m^{\prime}+n^{\prime}\right)=(C) \int f d(\alpha m+\beta n)=(C) \int f d(\alpha m)+$ $(C) \int f d(\beta n)=x_{1}+x_{2}$, hence $f \in \mathcal{L}_{A C}(\alpha \mu+\beta v)$.

Moreover, using Theorems 3.2 and 3.5 we obtain that $(C) \int f d\left(m^{\prime}+\right.$ $\left.n^{\prime}\right)=(C) \int f d(\alpha m+\beta n)=\alpha(C) \int f d m+\beta(C) \int f d n \in a(A C) \int f d \mu+$ 
$\beta(A C) \int f d v$, hence $(A C) \int f d(\alpha \mu+\beta v) \subset a(A C) \int f d \mu+\beta(A C) \int f d v$. Conversely, let be $z \in a(A C) \int f d \mu+\beta(A C) \int f d v$. Then we can write $z=z_{1}+z_{2}$, where $z_{1} \in a(A C) \int f d \mu$ and $z_{2} \in \beta(A C) \int f d v$. Hence there exists $m_{1}^{\prime} \in S_{\mu}^{C}(f)$ and $n_{1}^{\prime} \in S_{v}^{C}(f)$ such that $z_{1}=a(C) \int f d m_{1}^{\prime}$, and $z_{2}=\beta(C) \int f d n_{1}^{\prime}$. From properties of real Choquet integral (Theorems 3.2, iv) and 3.5, i)) we obtain that

$$
\begin{aligned}
z & =z_{1}+z_{2}=(C) \int f d\left(\alpha m_{1}^{\prime}\right)+(C) \int f d\left(\beta n_{1}^{\prime}\right) \\
& =\int_{0}^{\infty}\left(\alpha m_{1}^{\prime}\right)(\{s: f(s) \geq t\}) d t+\int_{0}^{\infty}\left(\beta n_{1}^{\prime}\right)(\{s: f(s) \geq t\}) d t \\
& =\int_{0}^{\infty}\left(\alpha m_{1}^{\prime}+\beta n_{1}^{\prime}\right)(\{s: f(s) \geq t\}) d t=(C) \int f d\left(\alpha m_{1}^{\prime}+\beta n_{1}^{\prime}\right)
\end{aligned}
$$

and hence $z \in(A C) \int f d(\alpha \mu+\beta v)$. Consequently we have that $(A C) \int f d(\alpha \mu$ $+\beta v)=a(A C) \int f d \mu+\beta(A C) \int f d v$.

Theorem 4.9. Let be $\mu: \mathcal{A} \rightarrow \mathcal{P}_{k c}\left(\mathbb{R}_{+}\right)$a multisubmeasure and $f, g:$ $S \rightarrow \mathbb{R}_{+}$two $A C$ - integrable functions with respect to $\mu$ on $S$. If $f$ and $g$ are comonotonic functions, then $f+g$ is $A C$ integrable and $(A C) \int(f+g) d \mu \subseteq$ $(A C) \int f d \mu+(A C) \int g d \mu$.

Proof. Since $f$ and $g$ are AC-integrable functions we have that $(A C) \int f d \mu=\left\{(C) \int f d m, m \in S_{\mu}^{C}(f)\right\} \neq \emptyset$ and $(A C) \int g d \mu=\left\{(C) \int g d n\right.$, $\left.n \in S_{\mu}^{C}(g)\right\} \neq \emptyset$. Firstly, we observe that $S_{\mu}^{C}(f+g)=S_{\mu}^{C}(f) \cap S_{\mu}^{C}(g)$.

Indeed, $S_{\mu}^{C}(f+g)=\left\{m \in S_{\mu}^{C}:(C) \int(f+g) d m<\infty\right\}$. But, since $f$ and $g$ are comonotonic functions then $(C) \int(f+g) d m=(C) \int f d m+(C) \int g d m<$ $\infty$.

Hence $(C) \int f d m<\infty$ and $(C) \int g d m<\infty$ and then $m \in S_{\mu}^{C}(f) \cap$ $S_{\mu}^{C}(g)$. Thus $S_{\mu}^{C}(f+g) \subseteq S_{\mu}^{C}(f) \cap S_{\mu}^{C}(g)$.

Analogously, we can prove the conversely inclusion.

Now, let be any $y_{0} \in(A C) \int(f+g) d \mu$. Then there exists $m \in S_{\mu}^{C}(f+g)$ such that we have $y_{0}=(C) \int(f+g) d m$. Since $f$ and $g$ are comonotonic functions then using Theorem 3.6 we obtain that $y_{0}=(C) \int(f+g) d m=$ $(C) \int f d m+(C) \int g d m=y_{1}+y_{2}$, where $y_{1}=(C) \int f d m \in(A C) \int f d \mu$ and $y_{2}=(C) \int g d m \in(A C) \int g d \mu$.

Hence, we have that $(A C) \int(f+g) d \mu \subseteq(A C) \int f d \mu+(A C) \int g d \mu$.

Proposition 4.10. Let be $\mu: \mathcal{A} \rightarrow \mathcal{P}_{k c}\left(\mathbb{R}_{+}\right)$a multisubmeasure and $f: S \rightarrow \mathbb{R}_{+}$an $A C$ - integrable function with respect to $\mu$ on $S$. Then $(A C) \int_{S} f d \mu$ is a convex set. 
Proof. Since $f$ is AC integrable with respect to $\mu$ on $S$ we have $(A C) \int_{S} f d \mu \neq \emptyset$. Let be $y_{1}, y_{2} \in(A C) \int_{S} f d \mu$, arbitrarily chosen, such that $y_{1}<y_{2}$. Then there exists $m, n \in S_{\mu}^{C}(f)$ such that $y_{1}=(C) \int f d m<\infty$ and $y_{2}=(C) \int f d n<\infty$. From Proposition 4.3, for every $\alpha \in(0,1)$ we have that $m^{\prime}=\alpha m+(1-\alpha) n \in S_{\mu}^{C}(f)$ and from Theorems 3.2, iv) and 3.3 we obtain

$$
\begin{aligned}
y & =(C) \int f d m^{\prime}=(C) \int f d(\alpha m+(1-\alpha) n) \\
& =(C) \int f d(\alpha m)+(C) \int f d((1-\alpha) n) \\
& =\alpha(C) \int f d m+(1-\alpha)(C) \int f d n=\alpha y_{1}+(1-\alpha) y_{2}<\infty .
\end{aligned}
$$

Then $y=\alpha y_{1}+(1-\alpha) y_{2} \in\left(y_{1}, y_{2}\right) \subset(A C) \int_{S} f d \mu$ and hence $(A C) \int_{S} f d \mu$ is a convex set.

Theorem 4.11. Let be $\mu: \mathcal{A} \rightarrow \mathcal{P}_{k c}\left(\mathbb{R}_{+}\right)$a multisubmeasure and $f: S \rightarrow$ $\mathbb{R}_{+}$a measurable function. If $f \in \mathcal{L}_{C}\left(m_{2}\right)$, where $m_{2}: \mathcal{A} \rightarrow \mathbb{R}_{+}$is defined by (1), then for every $A \in \mathcal{A}, f \in \mathcal{L}_{A C}(\mu, A)$ and

$$
(A C) \int_{A} f d \mu \subseteq\left[(C) \int_{A} f d m_{1},(C) \int_{A} f d m_{2}\right] .
$$

Proof. Let be $A \in \mathcal{A}$ fixed. From Remark 2.20, since $\mu(A)=\left[m_{1}(A)\right.$, $\left.m_{2}(A)\right]$ then for every $m: \mathcal{A} \rightarrow \mathbb{R}_{+}$with $m \in S_{\mu}$ we have

$$
m_{1}(A) \leq m(A) \leq m_{2}(A) .
$$

Since $f \in \mathcal{L}_{C}\left(m_{2}\right)$, then $f \in \mathcal{L}_{C}\left(m_{2}, A\right)$, and hence $(C) \int_{A} f d m_{2}<\infty$. From (5) we have that $(C) \int_{A} f d m<\infty$ and hence $(A C) \int_{A} f d \mu \neq \emptyset$. Obviously, for every $m \in S_{\mu}^{C}(f)$ we have $\int_{0}^{\infty} m_{1}(\{s: f(s) \geq t\} \cap A) d t \leq \int_{0}^{\infty} m(\{s:$ $f(s) \geq t\} \cap A) d t \leq \int_{0}^{\infty} m_{2}(\{s: f(s) \geq t\} \cap A) d t$ and from $\int_{0}^{\infty} m(\{s: f(s) \geq$ t\} $\cap A) d t=(C) \int_{A} f d m \in(A C) \int_{A} f d \mu$ we obtain that $(A C) \int_{A} f d \mu \subseteq$ $\left[(C) \int_{A} f d m_{1},(C) \int_{A} f d m_{2}\right]$

If $m: \mathcal{A} \rightarrow \mathbb{R}_{+}$is a strict submeasure then we proved in [21], ex. 2.15 that $\mu: \mathcal{A} \rightarrow P_{k c}\left(\mathbb{R}_{+}\right)$defined by $\mu(A)=[0, m(A)]$ is a strict multisubmeasure, called the strict multisubmeasure induced by $m$.

Now, we can easily obtain the following: 
Corollary 4.12. Let be $m: A \rightarrow \mathbb{R}_{+}$a strict submeasure, $\mu: A \rightarrow P_{k c}\left(\mathbb{R}_{+}\right)$ the strict multisubmeasure induced by the submeasure $m$ and $f: S \rightarrow \mathbb{R}_{+} a$ measurable function.

If $f \in L_{C}(m)$, then $f \in L_{A C}(\mu)$ and $(A C) \int_{A} f d \mu \subseteq\left[0,(C) \int_{A} f d m\right]$, for every $A \in \mathcal{A}$.

Proposition 4.13. Let be $\mu: \mathcal{A} \rightarrow \mathcal{P}_{k c}\left(\mathbb{R}_{+}\right)$a multisubmeasure and $f, g:$ $S \rightarrow \mathbb{R}_{+}$measurable functions such that $f, g \in \mathcal{L}_{C}\left(m_{2}\right)$, where $m_{2}$ is defined by (1). If $f \leq g$ on $S$ then, for every $A \in \mathcal{A}$, we have that $(A C) \int_{A} f d \mu \lesssim$ $(A C) \int_{A} g d \mu$.

Proof. Since $f \in \mathcal{L}_{C}\left(m_{2}\right)$ from Theorem 4.11 we have that, for every $A \in \mathcal{A}, f \in \mathcal{L}_{A C}(\mu, A)$ and

$$
(A C) \int_{A} f d \mu \subseteq\left[(C) \int_{A} f d m_{1},(C) \int_{A} f d m_{2}\right] .
$$

Analogously we obtain from $g \in \mathcal{L}_{C}\left(m_{2}\right)$ that $g \in \mathcal{L}_{A C}(\mu, A)$ and

$$
(A C) \int_{A} g d \mu \subseteq\left[(C) \int_{A} g d m_{1},(C) \int_{A} g d m_{2}\right] .
$$

Since $f \leq g$ on $S$ we obtain from Theorem 3.3 that for every $A \in \mathcal{A}$ $(C) \int_{A} f d m_{1} \leq(C) \int_{A} g d m_{1}$ and $(C) \int_{A} f d m_{2} \leq(C) \int_{A} g d m_{2}$. Then from Proposition 2.3 we have that $\left[(C) \int_{A} f d m_{1},(C) \int_{A} f d m_{2}\right] \lesssim\left[(C) \int_{A} g d m_{1}\right.$, (C) $\left.\int_{A} g d m_{2}\right]$.

Using Lemma 2.21 we obtain that $(A C) \int_{A} f d \mu \lesssim(A C) \int_{A} g d \mu$, for every $A \in \mathcal{A}$.

Theorem 4.14. Let be $\mu: \mathcal{A} \rightarrow \mathcal{P}_{k c}\left(\mathbb{R}_{+}\right)$a multisubmeasure and $f:$ $S \rightarrow \mathbb{R}_{+}$a measurable functions such that $f \in \mathcal{L}_{C}\left(m_{2}\right)$, where $m_{2}$ is defined by (1). Then, for every $A, B \in \mathcal{A}$ with $A \subseteq B$ we have $(A C) \int_{A} f d \mu \lesssim$ $(A C) \int_{B} f d \mu$.

Proof. Since $f \in \mathcal{L}_{C}\left(m_{2}\right)$ then for $A, B \in \mathcal{A}$ with $A \subseteq B$ we have that $f \in \mathcal{L}_{C}\left(m_{2}, A\right)$, respectively $f \in \mathcal{L}_{C}\left(m_{2}, B\right)$. From $(C) \int_{A} f d m_{1} \leq$ $(C) \int_{A} f d m_{2}<\infty$, respectively from $(C) \int_{B} f d m_{1} \leq(C) \int_{B} f d m_{2}<\infty$, we obtain that $f \in \mathcal{L}_{C}\left(m_{1}, A\right)$ and $f \in \mathcal{L}_{C}\left(m_{1}, B\right)$. Then, since $f \in$ $\mathcal{L}_{C}\left(m_{1}, A\right) \cap \mathcal{L}_{C}\left(m_{2}, A\right)$ and $f \in \mathcal{L}_{C}\left(m_{1}, B\right) \cap \mathcal{L}_{C}\left(m_{2}, B\right)$ we have, from Theorem 4.11, that $f \in \mathcal{L}_{A C}(\mu, A)$ and $f \in \mathcal{L}_{A C}(\mu, B)$ and

$$
(A C) \int_{A} f d \mu \subseteq\left[(C) \int_{A} f d m_{1},(C) \int_{A} f d m_{2}\right],
$$


respectively

$$
(A C) \int_{B} f d \mu \subseteq\left[(C) \int_{B} f d m_{1},(C) \int_{B} f d m_{2}\right]
$$

Since $A \subseteq B$, from Theorem 3.2, vi) we obtain that $(C) \int_{A} f d m_{1} \leq(C) \int_{B} f d m_{1}$ and $(C) \int_{A} f d m_{2} \leq(C) \int_{B} f d m_{2}$.

From Proposition 2.3 we easily see that

$$
\left[(C) \int_{A} f d m_{1},(C) \int_{A} f d m_{2}\right] \lesssim\left[(C) \int_{B} f d m_{1},(C) \int_{B} f d m_{2}\right]
$$

Using (8), (9), (10) and Lemma 2.21 we obtain that for every $A, B \in \mathcal{A}$ with $A \subseteq B$ we have $(A C) \int_{A} f d \mu \lesssim(A C) \int_{B} f d \mu$.

Proposition 4.15. Let be $\mu, \nu: \mathcal{A} \rightarrow P_{k c}\left(\mathbb{R}_{+}\right)$multisubmeasures such that $\mu(A)=\left[m_{1}(A), m_{2}(A)\right]$ and $\nu(A)=\left[n_{1}(A), n_{2}(A)\right]$, for every $A \in \mathcal{A}$, where $m_{1}, m_{2}, n_{1}, n_{2}$ are defined by (1). If $f: S \rightarrow \mathbb{R}_{+}$is a measurable function such that $f \in L_{C}\left(m_{2}\right)$ and $f \in L_{C}\left(n_{2}\right)$, then the following statements hold:

i) if $f \in \mathcal{L}_{C}\left(m_{2} \vee n_{2}\right)$ then $(A C) \int_{A} f d \mu \cup(A C) \int_{A} f d \nu \lesssim(A C) \int_{A} f d(\mu \vee$ $\nu)$, for every $A \in \mathcal{A}$;

ii) if $f \in \mathcal{L}_{C}\left(m_{2} \wedge n_{2}\right)$ then $(A C) \int_{A} f d(\mu \wedge \nu) \lesssim(A C) \int_{A} f d \mu \cap(A C) \int_{A} d \nu$, for every $A \in \mathcal{A}$.

Proof. Firstly, let us observe that since for every $A \in \mathcal{A}, \mu(A)=$ $\left[m_{1}(A), m_{2}(A)\right], \nu(A)=\left[n_{1}(A), n_{2}(A)\right]$ we have from Definition 2.22 that $(\mu \vee \nu)(A)=\mu(A) \vee \nu(A)=\left[m_{1}(A) \vee n_{1}(A), m_{2}(A) \vee n_{2}(A)\right]$, respectively $(\mu \wedge \nu)(A)=\mu(A) \wedge \nu(A)=\left[m_{1}(A) \wedge n_{1}(A), m_{2}(A) \wedge n_{2}(A)\right]$.

Let be $A \in \mathcal{A}$ arbitrarily chosen.To prove i) let us observe that since $f \in \mathcal{L}_{C}\left(m_{2}\right)$ from Theorem 4.11 we have $f \in \mathcal{L}_{A C}(\mu, A)$ and $(A C) \int_{A} f d \mu \subseteq$ $\left[(C) \int_{A} f d m_{1},(C) \int_{A} f d m_{2}\right]$. Analogously from $f \in \mathcal{L}_{C}\left(n_{2}\right)$ we obtain that $f \in \mathcal{L}_{A C}(\mu, A)$ and $(A C) \int_{A} f d \nu \subseteq\left[(C) \int_{A} f d n_{1},(C) \int_{A} f d n_{2}\right]$, for every $A \in \mathcal{A}$. Then $(A C) \int_{A} f d \mu \cup(A C) \int_{A} f d \nu \subseteq\left[(C) \int_{A} f d m_{1} \wedge(C) \int_{A} f d n_{1}\right.$, (C) $\left.\int_{A} f d m_{2} \vee(C) \int_{A} f d n_{2}\right]$

Let be any $y_{0} \in(A C) \int_{A} f d(\mu \vee \nu)$. Then there exists $m \in S_{\mu \vee \nu}^{C}(f)$ such that $y_{0}=(C) \int_{A} f d m$.

Since $m \in S_{\mu \vee \nu}^{C}(f)$ we have $m_{1} \vee n_{1} \leq m \leq m_{2} \vee n_{2}$. 
Because $m_{1} \leq m_{1} \vee n_{1}, m_{1} \in S_{\mu}^{C}(f)$ we obtain

$$
x_{0}=(C) \int_{A} f d m_{1} \in(A C) \int_{A} f d \mu \cup(A C) \int_{A} f d \nu
$$

and from Theorem 3.5, iii) we have $x_{0}=(C) \int_{A} f d m_{1} \leq(C) \int_{A} f d m=y_{0}$.

Hence, the condition i) from Definition 2.2 is true. Analogously we can prove ii) from Definition 2.2 and so we have that $(A C) \int_{A} f d \mu \cup(A C) \int_{A} f d \nu \lesssim$ $(A C) \int_{A} f d(\mu \vee \nu)$, for every $A \in \mathcal{A}$, and hence i) is true.

Analogously, we can prove ii).

\section{Some properties of the multifunction defined as an Au- mann-Choquet integral}

In this section we shall prove that for $\mu: \mathcal{A} \rightarrow \mathcal{P}_{k c}\left(\mathbb{R}_{+}\right)$a multisubmeasure and $f: S \rightarrow \mathbb{R}_{+}$an AC-integrable function if we impose supplementary conditions to $\mu$, the same properties are also preserved by the multifunction $I$ defined by

$$
I(E)=(A C) \int_{E} f d \mu, E \in \mathcal{A} .
$$

From Theorem 4.15 we can easily obtain:

Proposition 5.1. The multifunction I defined by (12) is a monotone set multifunction.

Proposition 5.2. Let be $\mu: \mathcal{A} \rightarrow \mathcal{P}_{k c}\left(\mathbb{R}_{+}\right)$a multisubmeasure and $f: S \rightarrow \mathbb{R}_{+}$a function such that $f \in \mathcal{L}_{A C}(\mu)$. If $\mu$ is weakly null-additive, then the multifunction defined by (12) is weakly null-additive.

Proof. Since $\mu$ is a weakly null-additive multisubmeasure then for every $A, B \in \mathcal{A}$ with $\mu(A)=\mu(B)=\{0\}$ we have $\mu(A \cup B)=\{0\}$.

Let be $A, B \in \mathcal{A}$ with $I(A)=I(B)=\{0\}$. Firstly, let us observe that every fuzzy measure selector $m$ of $\mu$ is weakly null-additive.

Indeed, since $\mu(A)=\left[m_{1}(A), m_{2}(A)\right]$, where $m_{1}, m_{2}$ are defined by (1) then, for every $A, B \in \mathcal{A}$ with $\mu(A)=\mu(B)=\{0\}$ we have $\left[m_{1}(A), m_{2}(A)\right]=$ $\{0\}$ and $\left[m_{1}(B), m_{2}(B)\right]=\{0\}$.

Hence $m_{1}(A)=0=m_{2}(A)$ and $m_{1}(B)=m_{2}(B)=0$ and for every $m \in S_{\mu}$ we have $m(A)=m(B)=0$. From $\mu(A \cup B)=\left[m_{1}(A \cup B), m_{2}(A \cup B)\right]=\{0\}$ we easily obtain that $m(A \cup B)=0$. Consequently, $m$ is null-additive. 
Since $f \in \mathcal{L}_{A C}(\mu)$ we have from Proposition 4.6 that $I(A \cup B)=$ $(A C) \int_{A \cup B} f d \mu=\left\{(C) \int_{A \cup B} f d m, m \in S_{\mu}^{C}(f)\right\} \neq \emptyset$.

Let be any $y \in I(A \cup B)$. Then there exists $m \in S_{\mu}^{C}(f)$, weakly nulladditive, such that $y=(C) \int_{A \cup B} f d m$.

Hence, from [24], th.7 we obtain that $y=(C) \int_{A \cup B} f d m=0$ and so $I(A \cup B)=\{0\}$, which assures that $I(\cdot)$ is weakly null-additive.

Proposition 5.3. Let be $\mu: \mathcal{A} \rightarrow \mathcal{P}_{k c}\left(\mathbb{R}_{+}\right)$a multisubmeasure and $f:$ $S \rightarrow \mathbb{R}_{+}$a function such that $f \in \mathcal{L}_{A C}(\mu)$. If $\mu$ is a null-additive multisubmeasure then the multifunction defined by (12) is null-additive.

Proof. We shall prove that if $A, B \in \mathcal{A}$ and $I(A)=\{0\}$ we obtain $I(A \cup B)=I(B)$. As in the proof of Proposition 5.2, we can show that if $\mu$ is null-additive then every $m \in S_{\mu}$ is a null-additive fuzzy measure. From $S_{\mu}^{C} \subseteq S_{\mu}$ we have that every $m \in S_{\mu}^{C}$ is null-additive.

Now, let be $x_{0} \in I(A \cup B)$ arbitrarily chosen. Then there exists $m^{\prime} \in S_{\mu}^{C}$, null-additive, such that $x_{0}=(C) \int_{A \cup B} f d m^{\prime}$.

Moreover, since $m^{\prime}$ is null-additive we obtain $x_{0}=(C) \int_{A \cup B} f d m^{\prime}=$ $\int_{0}^{\infty} m^{\prime}(\{s: f(s) \geq t\} \cap(A \cup B)) d t=\int_{0}^{\infty} m^{\prime}(\{s: f(s) \geq t\} \cap B) d t=$ $(C) \int_{B} f d m^{\prime} \in I(B)$, which assures that $I(A \cup B) \subseteq I(B)$.

Analogously, we can prove the converse inclusion. Indeed, if $y_{0} \in$ $I(B)$ is arbitrarily chosen then there exists $m^{\prime \prime} \in S_{\mu}^{C}$, null-additive such that $y_{0}=(C) \int_{B} f d m^{\prime \prime}$. Since $m^{\prime \prime}$ is null-additive then we have that $y_{0}=$ (C) $\int_{B} f d m^{\prime \prime}=\int_{0}^{\infty} m^{\prime \prime}(\{s: f(s) \geq t\} \cap B) d t=\int_{0}^{\infty} m^{\prime \prime}(\{s: f(s) \geq t\} \cap(A \cup$ $B) d t=(C) \int_{A \cup B} f d m^{\prime \prime}$, hence $y_{0} \in I(A \cup B)$ and the proof is completed. $\square$

In what follows we shall prove that if we impose supplementary conditions for the multisubmeasure $\mu$, such as converse null-additivity, pseudo generating property or Darboux property, then they are also preserved by the corresponding Aumann-Choquet integral.

Indeed we have:

Proposition 5.4. Let be $\mu: \mathcal{A} \rightarrow \mathcal{P}_{k c}\left(\mathbb{R}_{+}\right)$a multisubmeasure such that every $m \in S_{\mu}$ is converse null-additive and $f: S \rightarrow \mathbb{R}_{+}$an $A C$ - integrable function with respect to $\mu$ on $S$. Then the multifunction defined by (12) is converse null-additive.

Proof. To prove that $I$ is converse null-additive let be any $A, B \in \mathcal{A}$ with $A \subset B$ and $I(A)=I(B)$.

Firstly, we prove that $\mu$ is converse null-additive. 
Indeed, since every $m \in S_{\mu}$ is a converse null-additive fuzzy measure, from $S_{\mu}^{C}(f) \subseteq S_{\mu}$ we obtain that every $m \in S_{\mu}^{C}(f)$ is converse null-additive. Since from (1) we have $\mu(E)=\left[m_{1}(E), m_{2}(E)\right]$, for every $E \in \mathcal{A}$, where $m_{1}, m_{2}$ are converse null-additive, if $\mu(A)=\mu(B)$ then $\left[m_{1}(A), m_{2}(A)\right]=$ $\left[m_{1}(B), m_{2}(B)\right]$ and thus $m_{i}(A)=m_{i}(B), i=1,2$. Because $m_{i}, i=1,2$, are converse null-additive we have $m_{1}(B-A)=0=m_{2}(B-A)$, and consequently $\mu(B-A)=\{0\}$ which assures that $\mu$ is a converse null-additive multisubmeasure.

From $f \in \mathcal{L}_{A C}(\mu)$ and Proposition 4.6 we have that $f \in \mathcal{L}_{A C}(\mu, B-A)$ and hence $I(B-A)=(A C) \int_{B-A} f d \mu=\left\{(C) \int_{B-A} f d m, m \in S_{\mu}^{C}(f)\right\} \neq \emptyset$. From [24], th.8, since $m$ is a converse null-additive fuzzy measure then the set function defined by $\nu(A)=(C) \int_{A} f d m, A \in \mathcal{A}$, is converse nulladditive. Hence $(C) \int_{B-A} f d m=0$, for every $m \in S_{\mu}^{C}(f)$, and we have that $I(B-A)=(A C) \int_{B-A} f d \mu=\{0\}$ and consequently $I$ is converse null-additive.

Proposition 5.5. Let be $\mu: \mathcal{A} \rightarrow \mathcal{P}_{k c}\left(\mathbb{R}_{+}\right)$a multisubmeasure and $f:$ $S \rightarrow \mathbb{R}_{+}$an AC-integrable function with respect to $\mu$ on $S$. If $\mu$ has the pseudometric generating property then the same is true for the multifunction defined by (12).

Proof. Firstly, let us observe that since $\mu$ has p.g.p then every fuzzy measure $m \in S_{\mu}$ has p.g.p. Indeed, since $\mu$ has p.g.p, then for every $\varepsilon>0$ there exists $\delta>0$ such that for every $A, B \in \mathcal{A}$ with $\mu(A) \subseteq[0, \delta]$ and $\mu(B) \subseteq[0, \delta]$ we have $\mu(A \cup B) \subseteq[0, \varepsilon]$. From (1) we have that $\mu(A)=$ $\left[m_{1}(A), m_{2}(A)\right] \subseteq[0, \delta]$ and $\mu(B)=\left[m_{1}(B), m_{2}(B)\right] \subseteq[0, \delta]$ and for every $m \in S_{\mu}$ we have $m(A) \leq m_{2}(A) \leq \delta$ and $m(B) \leq m_{2}(B) \leq \delta$. From $\mu(A \cup B)=\left[m_{1}(A \cup B), m_{2}(A \cup B)\right] \subseteq[0, \varepsilon]$ we obtain that $m(A \cup B) \leq$ $m_{2}(A \cup B) \leq \varepsilon$, hence $m \in S_{\mu}$ has p.g.p.

Since $S_{\mu}^{C}(f) \subseteq S_{\mu}$, then every $m \in S_{\mu}^{C}(f)$ has p.g.p. Now, for every $\varepsilon>0$ we consider $\delta>0$ from the definition of p.g.p. of $\mu$ and let be $A, B \in \mathcal{A}$ with $I(A) \subseteq[0, \delta]$ and $I(B) \subseteq[0, \delta]$. Let be any $y \in I(A \cup B)$, where $I(A \cup B)=(A C) \int_{A \cup B} f d \mu=\left\{(C) \int_{A \cup B} f d m, m \in S_{\mu}^{C}(f)\right\}$. Then there exists $m \in S_{\mu}^{C}(f)$ with p.g.p. such that $y=(C) \int_{A \cup B} f d m$ and from [20], th.5 the set function defined as Choquet integral has p.g.p. and hence we obtain that $y \leq \varepsilon$.

Hence $I(A \cup B)=(A C) \int_{A \cup B} f d \mu \subseteq[0, \varepsilon]$ and $I$ has p.g.p.

Theorem 5.6. Let be $\mu: \mathcal{A} \rightarrow \mathcal{P}_{k c}\left(\mathbb{R}_{+}\right)$a multisubmeasure and $f: S \rightarrow$ $\mathbb{R}_{+}$a function $A C$ - integrable with respect to $\mu$ on $S$ such that $f(s) \neq 0$, 
for every $s \in S$. If every $m \in S_{\mu}$ has the Darboux property, then the multifunction defined by (12) has the Darboux property.

Proof. Since $f \in \mathcal{L}_{A C}(\mu)$ we have from Proposition 4.6, that $I(A)=$ $(A C) \int_{A} f d \mu \neq \emptyset$, for every $A \in \mathcal{A}$. Let be any $m \in S_{\mu}^{C}(f)$ with Darboux property.

Since $m(A) \neq 0$ and $f(s) \neq 0$, for every $s \in S$ we have $I(A)=$ $(A C) \int_{A} f d \mu \nsupseteq\{0\}$. We shall prove that for any $p \in(0,1)$ there exists $B \in \mathcal{A}, B \subset A$ such that $I(B)=p I(A)$.

Let be any $y_{0} \in I(A)$. Then there exists $m^{\prime} \in S_{\mu}^{C}(f)$ with Darboux property, such that $y_{0}=(C) \int_{A} f d m^{\prime}=\int_{0}^{\infty} m^{\prime}(\{s: f(s) \geq t\} \cap A) d t$.

Since $m^{\prime}$ has Darboux property then for any $p \in(0,1)$ there exists $B \subset A$ such that $m^{\prime}(B)=p m^{\prime}(A)$.

Thus, we have that $y_{0}=\int_{0}^{\infty} m^{\prime}(\{s: f(s) \geq t\} \cap A) d t=\int_{0}^{\infty} \frac{1}{p} m^{\prime}(\{s:$ $f(s) \geq t\} \cap B) d t$ and so $y_{0}=\frac{1}{p} y_{o}^{\prime}$, where $y_{0}^{\prime} \in I(B)$. Consequently, we have $p I(A) \subseteq I(B)$. It is easy to see that $I(B) \subseteq p I(A)$, hence the multifunction defined by (12) has the Darboux property.

In what follows, we prove some absolutely continuity properties for the multifunction $I$ with respect to $\mu$.

Proposition 5.7. If $\mu: \mathcal{A} \rightarrow \mathcal{P}_{k c}\left(\mathbb{R}_{+}\right)$is a multisubmeasure and $f:$ $S \rightarrow \mathbb{R}_{+}$is an $A C$ - integrable function with respect to $\mu$ on $S$ then $I \ll_{I} \mu$, where $I(A)=(A C) \int_{A} f d \mu$, for every $A \in \mathcal{A}$.

Proof. Let be any $A \in \mathcal{A}$ with $\mu(A)=\{0\}$. Then we have $\mu(A)=$ $\left[m_{1}(A), m_{2}(A)\right]=\{0\}$ and, consequently, $m_{1}(A)=0$ and $m_{2}(A)=0$.

Then, for every $m \in S_{\mu}^{C}$, we have $m(A)=0$ and we obtain that $(A C) \int_{A} f d \mu=\left\{(C) \int_{A} f d m, m \in S_{\mu}^{C}(f)\right\}=\{0\}$, hence $I \ll_{I} \mu$.

Remark 5.8. From Remark 2.17 and Proposition 5.3 we obtain that if $\mu$ is a null-additive multisubmeasure then $I \ll_{I} \mu \Leftrightarrow I \ll_{I I} \mu$ where $I$ is given by (12).

Hence we have:

Proposition 5.9. If $\mu: \mathcal{A} \rightarrow \mathcal{P}_{k c}\left(\mathbb{R}_{+}\right)$is a null-additive multisubmeasure and $f: S \rightarrow \mathbb{R}_{+}$is an $A C$ - integrable function with respect to $\mu$ on $S$ then $I \ll_{I I} \mu$, where $I(A)=(A C) \int_{A} f d \mu$, for every $A \in \mathcal{A}$.

Denoting by $\gamma$ the fuzzy measure defined by $\gamma(A)=|\mu(A)|$, for every $A \in \mathcal{A}$ we have: 
Proposition 5.10. If $\mu: \mathcal{A} \rightarrow \mathcal{P}_{k c}\left(\mathbb{R}_{+}\right)$is a multisubmeasure and $f:$ $S \rightarrow \mathbb{R}_{+}$is a measurable function such that $f \in \mathcal{L}_{C}(\gamma)$, then $I \ll_{I V} \gamma$, where $I$ is the multifunction given by $I(A)=(A C) \int_{A} f d \mu$, for every $A \in \mathcal{A}$.

Proof. According to the Definition 2.16 we shall prove that for every $\varepsilon>0$ there exists $\delta>0$ such that for any $A \in \mathcal{A}$ with $\gamma(A)<\delta$ we have $I(A) \subseteq[0, \varepsilon]$.

Let be $A \in \mathcal{A}$, arbitrarily chosen. From hypothesis we obtain that $f \in \mathcal{L}_{C}\left(m_{2}, A\right)$ and from Theorem 4.11, $f$ is (AC)-integrable with respect to $\mu$ on $A$. From $f \in \mathcal{L}_{C}\left(m_{2}\right)$ we have $(C) \int f d m_{2}=\int_{0}^{\infty} m_{2}(\{s: f(s) \geq$ t\}) $d t<\infty$ and from [24], th.13, [4], th.4.4, for every $\varepsilon>0$ there exists $0<a<b$ such that

$$
\int_{0}^{a} m_{2}(\{s: f(s) \geq t\}) d t+\int_{b}^{\infty} m_{2}(\{s: f(s) \geq t\}) d t<\frac{\varepsilon}{2} .
$$

Let us consider $\delta=\frac{\varepsilon}{2(b-a)}$ and $\gamma(A)<\delta$. Then $\gamma(A)=|\mu(A)|=$ $m_{2}(A)<\delta$. To prove that $I(A) \subset[0, \varepsilon]$ let be any $y \in I(A)=(A C) \int_{A} f d \mu=$ $\left\{(C) \int_{A} f d m, m \in S_{\mu}^{C}(f)\right\}$. Then there exists $m \in S_{\mu}^{C}(f)$ such that $y=$ (C) $\int_{A} f d m$ and $m(A) \leq m_{2}(A)$. We obtain

$$
\begin{aligned}
y & =(C) \int_{A} f d m=\int_{0}^{\infty} m(\{s: f(s) \geq t\} \cap A) d t \\
& \leq \int_{0}^{\infty} m_{2}(\{s: f(s) \geq t\} \cap A) d t .
\end{aligned}
$$

From $\int_{0}^{\infty} m_{2}(\{s: f(s) \geq t\} \cap A) d t=\int_{0}^{a} m_{2}(\{s: f(s) \geq t\} \cap A) d t+\int_{a}^{b} m_{2}(\{s:$ $f(s) \geq t\} \cap A) d t+\int_{b}^{\infty} m_{2}(\{s: f(s) \geq t\} \cap A) d t$ and using (14) we have that $y=(C) \int_{A} f d m<\frac{\varepsilon}{2}+\int_{a}^{b} m_{2}(\{s: f(s) \geq t\} \cap A) d t<\frac{\varepsilon}{2}+\delta(b-a)=$ $\frac{\varepsilon}{2}+\frac{\varepsilon}{2(b-a)}(b-a)=\varepsilon$.

Hence, for every $y \in I(A)$ we have $y<\varepsilon$, that is $I(A) \subset[0, \varepsilon]$ and $I \ll_{I V} \gamma$.

Proposition 5.11. Let be $\mu: \mathcal{A} \rightarrow \mathcal{P}_{k c}\left(\mathbb{R}_{+}\right)$a multisubmeasure and $f:$ $S \rightarrow \mathbb{R}_{+}$a measurable function such that $f \in \mathcal{L}_{C}(\gamma)$. If the multisubmeasure $\mu$ is o-continuous then, $I \ll_{I I I} \gamma$ where $I$ is defined by (12).

Proof. We shall prove that for any $\left(A_{n}\right)_{n} \subset \mathcal{A}$ with $\gamma\left(A_{n}\right) \rightarrow 0$ we have $\left|I\left(A_{n}\right)\right| \rightarrow 0$. Since for every $A \in \mathcal{A}$ we have that $\mu(A)=\left[m_{1}(A), m_{2}(A)\right]$ then $\mu\left(A_{n}\right)=\left[m_{1}\left(A_{n}\right), m_{2}\left(A_{n}\right)\right]$. Because $\gamma\left(A_{n}\right)=\left|\mu\left(A_{n}\right)\right|=m_{2}\left(A_{n}\right) \rightarrow 0$ 
and since $m_{1}\left(A_{n}\right) \leq m_{2}\left(A_{n}\right), \forall n \in \mathbb{N}$ we have that $m_{1}\left(A_{n}\right) \rightarrow 0$. From [24], th.3,4 we have that $\nu_{1}\left(A_{n}\right) \rightarrow 0$ and $\nu_{2}\left(A_{n}\right) \rightarrow 0$, where we denote by $\nu_{i}(A)=(C) \int_{A} f d m_{i}, i=1,2$.

Since $f \in \mathcal{L}_{C}(\gamma)$ we easily obtain that $f \in \mathcal{L}_{C}\left(m_{1}\right)$ and from Theorem 4.11 we have that $f \in \mathcal{L}_{A C}\left(\mu, A_{n}\right)$ and

$$
(A C) \int_{A_{n}} f d \mu \subseteq\left[(C) \int_{A_{n}} f d m_{1},(C) \int_{A_{n}} f d m_{2}\right] \text {, for every } n \in \mathbb{N} .
$$

From (15) we have $h\left(I\left(A_{n}\right),\{0\}\right) \leq h\left(\left[(C) \int_{A_{n}} f d m_{1},(C) \int_{A_{n}} f d m_{2}\right],[0,0]\right)=$ $\max \left\{(C) \int_{A_{n}} f d m_{1},(C) \int_{A_{n}} f d m_{2}\right\}=(C) \int_{A_{n}} f d m_{2} \rightarrow 0$.

Hence $h\left(I\left(A_{n}\right),\{0\}\right) \rightarrow 0$ and we have $I \ll_{I I I} \gamma$.

Acknowledgement. The author is indebted to Prof. Dr. A.M. Precupanu for the careful reading of the paper and for the useful suggestions.

\section{REFERENCES}

1. Aumann, R.J. - Integrals of set-valued functions, J. Math. Anal. Appl., 12 (1965), $1-12$.

2. Choquet, G. - Theory of capacities, Ann. Inst. Fourier, Grenoble 5 (1953-1954), 131-295 (1955).

3. Guo, C.; Zhang, D. - On set-valued fuzzy measures, Inform. Sci., 160 (2004), 13-25.

4. Hongxia, S.; Jun, L.; Yasuda, M. - Monotone set-valued functions defined by setvalued Choquet integrals, Tenth International Conference on Fuzzy Set Theory and Applications, 2010.

5. Hu, S.; PApageorgiou, N.S. - Handbook of Multivalued Analysis, Vol. I, Theory, Mathematics and its Applications, 419, Kluwer Academic Publishers, Dordrecht, 1997.

6. JANG, L.C. - Interval-valued Choquet integrals and their applications, J. Appl. Math. and Computing, 16 (2004), 429-443.

7. JANG, L.-C. - A note on the monotone interval-valued set function defined by the interval-valued Choquet integral, Commun. Korean Math. Soc., 22 (2007), 227-234.

8. JANG, L.C.; KIM, T.; JeON, J. - On set-valued Choquet integrals and convergence theorems, Adv. Stud. Contemp. Math. (Kyungshang), 6 (2003), 63-76.

9. JANG, L.C.; KIM, T.K.; JEON, J.D. - On set-valued Choquet integrals and convergence theorems. II, Bull. Korean Math. Soc., 40 (2003), 139-147. 
10. JANG, L.C.; KwOn, J.S. - On the representation of Choquet integrals of set-valued functions, and null sets, Fuzzy Sets and Systems, 112 (2000), 233-239.

11. JAng, L.C.; KIL, B.M.; KIM, Y.K.; Kwon, J.S. - Some properties of Choquet integrals of set-valued functions, Fuzzy Sets and Systems, 91 (1997), 95-98.

12. KIM, H.M.; JANG, L.-C.; KIM, T. - On closed set-valued Choquet integrals with respect to non-monotonic fuzzy measures, Proc. Jangjeon Math. Soc., 7 (2004), 109116.

13. Klein, E.; Thompson, A.C. - Theory of Correspondences. Including Applications to Mathematical Economics, Canadian Mathematical Society Series of Monographs and Advanced Texts, A Wiley-Interscience Publication, John Wiley \& Sons, Inc., New York, 1984.

14. Murofushi, T.; Sugeno, M. - A theory of fuzzy measures: representations, the Choquet integral, and null sets, J. Math. Anal. Appl., 159 (1991), 532-549.

15. Murofushi, T.; Sugeno, M. - An interpretation of fuzzy measures and the Choquet integral as an integral with respect to a fuzzy measure, Fuzzy Sets and Systems, 29 (1989), 201-227.

16. PAP, E. - Null-Additive Set Functions, Mathematics and its Applications, 337, Kluwer Academic Publishers Group, Dordrecht; Ister Science, Bratislava, 1995.

17. Precupanu, A.-M.; Croitoru, A. - A Gould type integral with respect to a multimeasure. I, An. Ştiinţ. Univ. "Al.I. Cuza" Iaşi. Mat. (N.S.), 48 (2002), 165-200 (2003).

18. Precupanu, A.M.; Croitoru, A. - A Gould type integral with respect to a multimeasure. II, An. Ştiinţ. Univ. "Al.I. Cuza” Iaşi. Mat. (N.S.), 49 (2003), 183-207.

19. Precupanu, A.-M.; Satco, B. - The Aumann-Gould integral, Mediterr. J. Math., 5 (2008), 429-441.

20. Ouyang, Y.; Li, J. - Some properties of monotone set functions defined by Choquet integral, J. Southeast Univ. (English Ed.), 19 (2003), 423-426.

21. Sofian-BocA, F.-N. - Another Gould type integral with respect to a multisubmeasure, An. Ştiinţ. Univ. "Al.I. Cuza" Iaşi. Mat. (N.S.), 57 (2011), 13-30.

22. Zhang, D.; Guo, C.; Liu, D. - Set-valued Choquet integrals revisited, Fuzzy Sets and Systems, 147 (2004), 475-485.

23. Zhang, D.L.; Wang, Z.X. - On set-valued fuzzy integrals, Fuzzy Sets and Systems, 56 (1993), 237-241.

24. WAng, Z.; KliR, G.J.; WANG, W. - Monotone set functions defined by Choquet integral, Fuzzy Sets and Systems, 81 (1996), 241-250.

Received: 15.III.2012

Accepted: 16.IV.2012
Faculty of Mathematics, "Alexandru Ioan Cuza" University of Iaşi, Carol I Blvd., no. 11, 700506, ROMANIA nicolesof@yahoo.com 